\title{
Potential Roles of Long Non-coding RNAs in Regulating Cell Proliferation, Invasion, and Metastasis in Ovarian Serous Cystadenocarcinoma
}

\author{
Mujie Kan \\ Jilin University \\ Housen Zhang \\ Chengya Dong \\ Beijing Tiantan Hospital \\ Tianmin Xu \\ Jilin University Second Hospital \\ Qi Li ( $\square$ liqibjtt@ccmu.edu.cn ) \\ Beijing Tiantan Hospital
}

China-Japan Union Hospital of Jilin University

\section{Research}

Keywords: Ovarian serous cystadenocarcinoma. Microarray. LncRNA. Functional analysis, Transcription factor

Posted Date: November 1st, 2021

DOI: https://doi.org/10.21203/rs.3.rs-770129/v2

License: (c) (i) This work is licensed under a Creative Commons Attribution 4.0 International License. Read Full License 


\section{Abstract}

Background: Ovarian serous cystadenocarcinoma (OSC) is the most common gynecological malignancy. Long non-coding RNAs (IncRNAs) are aberrantly expressed in many cancers and involved in cell proliferation, apoptosis, angiogenesis, and invasion. Here, we investigated the functional roles of IncRNAs in OSC in detail.

Methods: We analyzed a cohort of exon microarray datasets from The Cancer Genome Atlas and used differentially expressed IncRNAs and mRNAs to construct an IncRNA-mRNA co-expression network. Distinct IncRNAs were classified into lincRNA, enhancer-like IncRNAs, or antisense IncRNAs. Biological functions for IncRNAs were predicted according to the IncRNA-mRNA network and genomic adjacency by KEGG pathway analysis. A transcription factor (TF)-IncRNA regulatory network was constructed by integrating IncRNA molecular profiles and TF binding information.

Results: We identified 2,939 IncRNAs and 2,766 mRNAs that were differentially expressed between OSC and normal ovary tissues. The 67 IncRNAs in the IncRNA-mRNA network, 23 lincRNAs, 19 antisense IncRNAs, and four enhancer-like IncRNAs were involved in cell proliferation, invasion, and metastasis. The TFs ING4, TTF-1, RUSH-I alpha, Kaiso, and STAT1 targeted regulation of IncRNAs in the pathological processes of OSC. Expression of 10 IncRNAs and mRNAs, as well as SOS1, ITGB1, and BIRC2 mRNAs with their identified IncRNAs were verified by qRT-PCR in OSC tissues.

Conclusions: We predicted the biological functions of many IncRNAs, which may serve as diagnostic and prognostic biomarkers as well as therapeutic targets in OSC.

\section{Introduction}

Ovarian cancer is the most lethal gynecologic malignancy in women, and ovarian serous cystadenocarcinoma (OSC) is the most common and aggressive subtype [1]. Although several methods are available for the management of OSC patients, including surgical resection, radiation therapy, chemotherapy and molecular-targeted therapy, most women diagnosed with OSC have a poor prognosis [2]. The development and progression of OSC are complex and involve a multiple-step process. Therefore, distinguishing the molecular characteristics of OSC from those of normal ovary tissues is pivotal for designing effective therapies that target tumor growth and metastasis. Moreover, new diagnostic and prognostic biomarkers are essential for extending the survival time of patients with ovarian cancer.

Long noncoding RNAs (IncRNAs) are non-protein coding RNAs > 200 nucleotides in length that play vital roles in various biological functions such as genetic imprinting, chromatin modification, and modulation of gene expression [3]. LncRNAs are critical regulators of cell biology whose alteration can lead to the development of diseases such as cancer [4]. Based on their location relative to protein coding genes, IncRNAs are classified as intergenic, intragenic, and antisense [5]. Large intergenic non-coding RNAs (lincRNAs), which do not overlap with protein coding regions, are key regulators of diverse cellular processes [6]. Enhancer-like IncRNAs are intergenic IncRNAs located in the enhancer region of the 
genome, and serve to activate gene expression in cis [7]. Antisense IncRNAs regulate the corresponding sense coding gene at the transcriptional or post-transcriptional level [8]. Although the IncRNA signatures of ovarian cancer have been established [9-12], most IncRNAs have not been functionally characterized.

In this study, we compared the expression of IncRNAs and mRNAs between eight OSC samples and eight normal ovarian tissues obtained from The Cancer Genome Atlas (TCGA) database. An IncRNA-mRNA coexpression network was constructed to reveal the functional role of IncRNAs in regulating tumor cell proliferation, invasion, and metastasis. Among the top differentially expressed IncRNAs, we identified lincRNAs, antisense IncRNAs, and enhancer IncRNAs associated with tumor growth and metastasis in OSC. Finally, OSC-specific transcription factor (TF)-IncRNA regulatory networks were constructed by integrating IncRNA expression profiles and TF binding information. TFs play a key role in controlling IncRNA expression. The identified sets of IncRNAs and mRNAs specific to OSC were confirmed by quantitative reverse transcription polymerase chain reaction (qRT-PCR) in OSC tissues.

\section{Materials And Methods}

\section{Dataset characteristics}

The Affymetrix Human Exon 1.0 array datasets of OSC were obtained from the TCGA database (https://tcga-data.nci.nih.gov/tcga/). LncRNA and mRNA expression signatures in OSC were analyzed using 16 datasets including eight non-tumor ovary samples and eight OSC samples.

Microarray data analysis

Two-class differential was used to determine the differentially expressed IncRNAs and mRNAs between the OSC and normal ovary groups. The random variance model (RVM) $t$ test was applied to filter the differentially expressed genes for the control and experimental groups, which can effectively increase the degree of freedom in cases of small samples. The fold-change $(F C)$ and $P$ values were calculated for each transcript between two comparison groups. $\mathrm{FC} \geq 2.0$ or $\leq 0.6$, and $P<0.05$ were the criteria to identify differentially expressed IncRNAs or mRNAs in OSC/normal ovary. The differentially expressed transcripts were imported into Cluster and TreeView (Stanford University) to perform hierarchical cluster analysis (HCA). The HCA grouped samples into transcript expression profiles according to their similarities.

Gene ontology and pathway analysis

The main functions of differentially expressed genes were determined by gene ontology (GO) analysis, which is the functional classification of NCBI. It can organize genes into hierarchical categories and uncover the gene regulatory networks on the basis of biological processes and molecular functions. The significant pathways of differentially expressed genes were determined by pathway analysis according to Kyoto Encyclopedia of Genes and Genomes (KEGG) (http://www.genome.jp/kegg/). Enrichment scoring, 
which corresponds to -log10 (P value), was used to measure function or pathway significance and specificity.

\section{Construction of the IncRNA-mRNA co-expression network}

The IncRNA-mRNA co-expression networks were built according to the normalized signal intensity of specific expression of mRNAs and IncRNAs. For each mRNA-IncRNA, mRNA-mRNA, or IncRNA-IncRNA pair, the Pearson's correlation was calculated and significant correlation pairs were selected to construct the network. The degree centrality is an important measure of the centrality of an mRNA or IncRNA within a network that determines its relative importance. Degree is defined as the number of connections of one node to the other. The clustering coefficient represents the density of each gene with the adjacent gene; the clustering coefficient of a gene is positively related to its importance in regulating the network.

When considering different networks, core regulatory factors were determined according to the degree of differences between two class samples. In two-network analysis, the degree values of the same mRNA/IncRNA in different networks were standardized, and the degree value of a certain mRNA/IncRNA was divided by the maximum degree in the network [Normalized degree (i) = Degree (i)/Degree (Max)].

The relative degree is expressed as tumor_K or normal_K. Next, we calculated the difference in relative degree between the tumor and normal groups to obtain the DiffK (tumor-normal). Finally, the results in the table were sorted by the absolute value |DiffK|.

Identification of transcription factor-IncRNA regulatory interactions

The sequences of differentially expressed IncRNAs near the transcription start site ( $1 \mathrm{~kb}$ upstream to 0.2 $\mathrm{kb}$ downstream) were searched, and the TRANSFACT database (Biobase) and the Match $^{\text {TM }}$ algorithm were used to predict the TFs of IncRNAs [13]. The search algorithm uses two score values: the matrix similarity score (MSS) and the core similarity score (CSS), which ranged from 0.0 to 1.0 , with 1.0 indicating an exact match. Next, the TF regulatory network was constructed using the interactions among IncRNAs and TFs (TF-IncRNA-network).

\section{Patient and tumor samples}

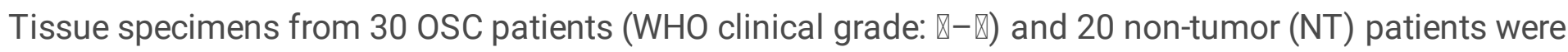
collected from the Department of Gynecology, The Second Hospital of Jilin University (Changchun, Jilin, China), between January 2013 and December 2014. Patients were included if they had not received chemotherapy or radiotherapy prior to surgery. Normal ovaries were obtained from 20 NT patients who underwent surgery for benign gynecological conditions. Samples were collected immediately after surgical resection, snap frozen, and stored at $-80^{\circ} \mathrm{C}$. Histological diagnoses were made using formalinfixed, paraffin-embedded hematoxylin and eosin sections, and reviewers were blinded to the original diagnosis according to the WHO classification. The use of patient samples was approved by the Ethics Committee of The Second Hospital of Jilin University. Written informed consent for the study was obtained from all subjects. 
Total RNA from various tissue specimens was extracted using the TRIzol reagent (Invitrogen, Carlsbad, CA, USA). RNA was reverse-transcribed with a PrimeScript ${ }^{\text {TM }}$ RT reagent kit (TaKaRa, Dalian, Liaoning, China) for CDNA synthesis and genomic DNA removal. The qPCR was performed using the TaKaRa realtime PCR system and SYBR® Premix Ex Taq ${ }^{\mathrm{TM}}$ II (TaKaRa). PCR reactions were performed using Platinum Taq DNA Polymerase Mixture (Invitrogen). The primers used are listed in Supplementary Table 1.

\section{Statistics}

For GO and pathway analysis, a two-sided Fisher's exact test was performed to classify the pathways, and $\mathrm{P}$ values were computed for the $\mathrm{GO}$ or pathway of each differentially expressed gene. GraphPad Prism 8.0 software (GraphPad Software Inc., La Jolla, CA, USA) was used for qRT-PCR statistical analyses. Data were presented as the mean \pm standard error of the mean (SEM). Significant differences were assessed by the Student's $t$-test for two-group comparisons. $P<0.05$ was considered statistically significant.

\section{Results}

\section{Distinctive IncRNA and mRNA expression between normal ovary and OSC tissues}

The IncRNA and mRNA expression patterns were compared between normal ovary tissues and OSC samples. Normal ovary tissues $(n=8)$ and OSC samples $(n=8)$ were subjected to the RVM $t$-test. A total of 2,939 IncRNAs and 2,766 mRNAs with significant differential expression between OSC and normal ovary tissues (fold change $\geq 2.0$ or $\leq 0.6, P<0.05$; Supplementary Tables $2 \& 3$ ) were identified. Of these 2,939 IncRNAs, 1,655 were downregulated and 1,284 were upregulated. The differences indicated their potential roles in OSC pathogenesis. Hierarchical cluster analysis showed systematic variations in the expression of differential IncRNAs and mRNAs in OSC and normal ovary tissues (Fig. 1a). Thus, the IncRNA and mRNA expression signatures were likely representative.

Construction of a IncRNA-mRNA co-expression network and prediction of IncRNA functional roles based on this network

A co-expression network was built to analyze the interactions between IncRNAs and mRNAs. The workflow of the construction of the IncRNA-mRNA co-expression network is shown in Fig. 1b. First, the main functions of the 2,766 differentially expressed mRNAs in OSC were determined by GO analysis. The results showed that 2,090 mRNAs were involved in 362 upregulated and 160 downregulated significant functions ( $P<0.05$; Supplementary Table 4). The pathways of the altered 2766 mRNAs involved were then analyzed according to KEGG. A total of 589 mRNAs were involved in the 59 enrichment-related pathways $(P<0.05$; Supplementary Table 5), 45 of which were upregulated and 14 were downregulated. 
As a result, 229 mRNAs (Supplementary Table 6) with significant functions and pathways in GO and pathway analysis were termed GO\&Pathway mRNAs. The levels of the GO\&Pathway mRNAs and the differentially expressed IncRNAs in the normal ovary and OSC groups were used to construct two IncRNAmRNA co-expression networks (Supplementary Figs. 1 \& 2). The mRNAs and IncRNAs involved in the two networks were sorted by degree and clustering coefficient as shown in Supplementary Tables $7 \& 8$.

To identify IncRNAs involved in OSC, the two IncRNA-mRNA co-expression networks were compared, and the difference in relative degree (|DiffK|) was calculated (Supplementary Table 9). The |DiffK| represents the change in the common expression status of IncRNAs in two networks. As listed in Supplementary Table 10, 67 IncRNAs (with a |DiffK| >0.25) were selected for further functional analysis.

According to the IncRNA-mRNA co-expression networks in the normal ovary and OSC groups, 109 mRNAs were co-expressed with the selected 67 IncRNAs. KEGG pathway analysis showed that the 109 mRNAs were involved in 32 upregulated and 4 downregulated pathways involved in various biological processes, including cellular processes, environmental information processing, human diseases, genetic information processing, metabolism, and immune system ( $P<0.05$; Fig. 2$)$.

Based on these pathways, we predicted the potential roles of the 67 IncRNAs in OSC pathogenesis. Coexpressed IncRNAs and mRNAs in upregulated pathways were shown in the phagosome, focal adhesion, apoptosis, cell cycle, regulation of actin cytoskeleton, progesterone-mediated oocyte maturation, ECMreceptor interaction, proteoglycans in cancer, pathways in cancer, PI3K-Akt, VEGF, p53, and oocyte meiosis signaling pathways (Fig. 3a, b; Supplementary Table 11); in the DNA replication, viral carcinogenesis, homologous recombination, mismatch repair, ribosome biogenesis in eukaryotes, RNA transport, nucleotide excision repair, proteasome, HIF-1, Fanconi anemia, and ubiquitin-mediated proteolysis signaling pathways (Fig. 3c, Supplementary Table 12); in thecytokine-cytokine receptor interaction, progesterone-mediated oocyte maturation, B cell receptor, chemokine, and Toll-like receptor signaling pathway (Fig. 3d, Supplementary Table 13); and in the purine metabolism, pyrimidine metabolism, tryptophan metabolism, biosynthesis of amino acids, and Metabolism pathways (Fig. 3e, Supplementary Table 14). Detailed descriptions of 28 mRNAs which participated in multiple pathways in the coexpression network are provided in Supplementary Table 15. The PIK3CA, KRAS, and NRAS genes play key roles in OSC progression. KRAS and NRAS function in seven signaling pathways; PIK3CA is involved in eleven signaling pathways, seven of which are common with KRAS and NRAS pathways. Co-expressed IncRNAs and mRNAs in downregulated pathways were involved in drug metabolism, lipid metabolism, tyrosine metabolism, and ABC transporter signaling pathways (Fig. 3f, Supplementary Table 16).

Taken together, these results demonstrated that IncRNA-regulated mRNAs were linked to multiple biological processes that affected tumor cell proliferation, apoptosis, migration, invasion, and angiogenesis.

Identification of the possible roles of IncRNAs based on genomic co-localization relative to protein coding genes 
To further explore the possible roles of differentially expressed IncRNAs during the development of OSC, we analyzed their genomic locations and classified them into lincRNA, antisense IncRNA, or enhancer-like IncRNA. Among the 2,939 differentially expressed IncRNAs between OSC and normal ovary tissues, 166 were lincRNAs, 144 were antisense IncRNAs, and 43 were enhancer-like IncRNAs. We combined these lincRNAs, antisense IncRNAs, and enhancer-like IncRNAs with their adjacent coding genes located within $300 \mathrm{~kb}$ in the genome and differentially expressed in OSC to analyze the potential functions of these IncRNAs. The IncRNAs and associated coding genes are shown in Supplementary Table 17.

LincRNAs are large intergenic non-coding RNAs (Fig. 4a). The functions of 144 lincRNAs were predicted through pathway analysis of adjacent genes. The results indicated that 23 lincRNAs may regulate the expression of 26 coding genes involved in 20 upregulated pathways (Fig. 4b). These pathways regulate cell adhesion, invasion, migration, proliferation, and apoptosis among cellular processes, genetic and environmental information processing, and metabolism (Fig. 4c-I). The coding genes SOS1 and ITGB1 may play a key role in tumor progression, and they were involved in more than six signaling pathways.

We then analyzed 144 antisense IncRNAs (Fig. 5a). KEGG pathway analysis was used to predict the biological roles of 19 antisense IncRNAs, and the results indicated that 16 coding genes were involved in 15 upregulated pathways and 2 downregulated pathways (Fig. 5b, c). These pathways are involved in cellular processes, metabolism, and environmental and genetic information processing. Of the 16 coding genes, RAD51, COL4A1, and BIRC2 were involved in more than one signaling pathway (Fig. 5d-j).

Finally, we predicted the roles of enhancer-like IncRNAs in OSC (Fig. 5K). Of 43 enhancer-like IncRNAs, four were positively correlated with the expression of five mRNAs. Therefore, the functions of four IncRNAs that acted as enhancers were predicted through pathway analysis of five coding genes. The results indicated that the coding genes $B M S 1$ and $A N A P C 1$ participated in four upregulated pathways (Fig. 5l). These pathways represent cellular processes and genetic information processing, including cell cycle, oocyte meiosis, ubiquitin-mediated proteolysis, and ribosome biogenesis in eukaryotes. The ubiquitin system regulates cell differentiation and immunity, and is involved in transcription, regulation of secretion, and cell development by mediating protein degradation. We predicted that two enhancer-like IncRNAs (n371668 and n378384) played important roles in OSC cell proliferation (Fig. 5m).

\section{Functional characterization of TF-IncRNA relationships in OSC}

To identify potential TF-IncRNA relationships, we constructed a TF-IncRNA-network and identified 1,034 potential TF-IncRNA interactions among 50 TFs and 100 differentially expressed IncRNAs (Fig. 6a, Supplementary Table 18). Next, we compared the mRNA expression patterns of 50 TFs between OSC and normal ovary tissues. The results indicated that ING4, TTF1, HLTF, ZBTB33, and STAT1 mRNAs (encoding the TFs ING4, TTF-1, RUSH-I alpha, Kaiso, and STAT1, respectively) were upregulated in OSC. Of the five TF-regulated IncRNAs, 37 IncRNAs (Supplementary Tables 11-14) were in the IncRNA-mRNA coexpression network and involved in several key signaling pathways. These five TFs and their target IncRNAs are shown in Fig. 6b. 


\section{The qRT-PCR analysis of the distinctive expression of IncRNAs and mRNAs in OSC samples}

To validate the results of microarray analysis, we randomly selected 10 IncRNAs and mRNAs with larger fold changes, including upregulation and downregulation, from the microarray results and analyzed their expression levels by qRT-PCR in 30 OSC tissues and 20 normal ovary tissues. The results showed that the changes were consistent between microarray and qPCR data, confirming the findings of the IncRNA and mRNA microarray data (Fig. 7a, b). Next, we analyzed the expression of SOS1 mRNA and lincRNA n365425, ITGB1 mRNA and lincRNA ENST00000450890, and BIRC2 mRNA and antisense IncRNAs ENST00000528717 and ENST00000544115 to explore whether SOS1, ITGB1, and BIRC2 were regulated by these differentially expressed IncRNAs in OSC. As shown in Fig. 7c, SOS1 mRNA was upregulated and lincRNA n365425 was downregulated, and ITGB1 mRNA and lincRNA ENST00000450890 were upregulated $(P<0.05)$. BIRC2 mRNA and antisense IncRNAs ENST00000544115 were upregulated, whereas antisense IncRNA ENST00000528717 was downregulated in OSC samples $(P<0.05)$.

\section{Discussion}

We used two strategies to predict the function of differentially expressed IncRNAs: one strategy was based on the construction of an IncRNA-mRNA co-expression network [14, 15], and the other one classified IncRNAs into lincRNAs, antisense IncRNAs, and enhancer IncRNAs and determined the genomic adjacency of differentially expressed mRNAs. We identified a set of 2,939 IncRNAs and 2,766 mRNAs with differential expression between OSC and normal ovary samples. This indicated that RNA homeostasis may be essential for tumor initiation and development in OSC, and its disruption could lead to changes in the biological function of tumor cells.

LncRNA-mRNA expression combined with KEGG pathway analysis identified 67 altered IncRNAs related to cell proliferation and apoptosis, migration and invasion, and amino acid and nucleotide metabolism. These IncRNAs were involved in 32 upregulated pathways and associated with four downregulated pathways, including tyrosine metabolism, drug metabolism-cytochrome P450, ether lipid metabolism, and $\mathrm{ABC}$ transporters.

A series of upregulated pathways indicated that OSC had strong proliferation and metastasis abilities and decreased apoptosis ability, suggesting that regulation of IncRNAs may be useful for the treatment of OSC. Moreover, our results suggested that differential expression of IncRNAs was involved in the regulation of amino acid metabolism in OSC. One upregulated pathway and one downregulated pathway were involved in the modulation of tryptophan metabolism and tyrosine metabolism. Changes in amino acid metabolism contribute to different aspects of tumorigenesis [16], and restoring these altered metabolic pathways may be a new approach to cancer treatment [17]. Previous reports suggested that the $A B C$ transporter pathway was inhibited in OSC; in this study, we confirmed that the previously identified $A B C A 8$ gene was significantly downregulated in ovarian cancer cells [18], and we extended 
these findings to IncRNA regulation. Moreover, the $A B C A 9$ and $A B C A 10$ genes showed decreased expression in OSC and were regulated by IncRNAs.

To explore possible mechanisms underlying the role of IncRNAs in regulating adjacent protein coding genes, we identified 23 lincRNAs, 19 antisense RNAs, and four enhancer-like IncRNAs that were differentially expressed and involved in modulating cell proliferation, adhesion, metastasis, invasion, and apoptosis in OSC by altering biological processes including environmental information processing, metabolism, genetic information processing, and cellular processes.

In this study, we showed that the coding genes PIK3CA, KRAS, and NRAS were involved in several pathways that modulated cell growth and death processes (focal adhesion; regulation of actin cytoskeleton; apoptosis), signal transduction (PI3K-Akt, HIF-1, VEGF signaling pathway), immune system (B cell receptor, chemokine, toll-like receptor signaling pathway) in OSC. KRAS and NRAS are different isoforms of the RAS gene; RAS is the most frequently mutated oncogene in human cancers, with mutations in approximately $30 \%$ of all cancers [19]. Somatic mutations in the PIK3CA gene were previously reported in ovarian cancer [20]. We observed that PIK3CA (fold change: 2.93), KRAS (fold change: 4.2), and NRAS (fold change: 5.21) were upregulated in OSC. Several IncRNAs including ENST00000537662, n343016, n324533, and n378874 were involved in the regulation of PIK3CA; other IncRNAs such as n324533, ENST00000469713, n342544, ENST00000489672, n341824, ENST00000509691, and ENST00000471635 modulated NRAS, and n341824 regulated KRAS.

This study confirmed many previously identified signature genes in tumors and extended these findings to IncRNA regulation. For example, SOS1, ITGB1, and BIRC2, which were upregulated in OSC compared with normal ovary tissues, were involved in multiple signaling pathways and regulated cell proliferation, apoptosis, and migration. Our findings suggested that SOS1 mRNA expression was regulated by lincRNA n365425, ITGB1 was regulated by lincRNA ENST00000450890, and BIRC2 was regulated by the antisense IncRNAs ENST00000528717 and ENST00000544115. Additionally, SOS1, ITGB1, and BIRC2 mRNAs and their regulatory IncRNAs in OSC and normal ovary tissues were detected by RT-qPCR. Taken together, these findings strongly suggested that SOS1, ITGB1, and BIRC2 gene expression was regulated by lincRNAs during tumor progression in OSC.

\section{Conclusions}

This study identified differentially expressed IncRNAs and mRNAs between OSC and normal ovary tissues, and explored the potential roles of these IncRNAs in regulating cell proliferation, invasion, and metastasis in OSC. The present data suggested that these dysregulated IncRNAs could serve as useful therapeutic targets and prognostic biomarkers in OSC.

\section{Declarations}

Acknowledgements 
The authors thank the Genminix Company (Shanghai, China) for assistance with bioinformatics analysis.

Author contributions

Study concept and design: Li Q and Xu TM. Acquisition of data: Kan MJ, Zhang HS and Dong CY. Analysis and interpretation of data: Kan MJ, Zhang HS and Dong CY. Drafting and editing of the manuscript: Kan MJ, Li Q and Xu TM. All authors contributed to the article and approved the submitted version.

Funding

This work was supported by the Health Technological Innovation Project of Jilin province of China (2019J012).

Availability of data and material

The dataset supporting the conclusions of this article is included within the article.

"The pre-print version of this article is present on https://www.researchsquare.com/article/rs-770129/v1. This article is not published nor is under publication elsewhere."

Ethics approval and consent to participate

The studies involving human participants were reviewed and approved by Ethics Committee of The Second Hospital of Jilin University. The patients provided their written informed consent to participate in this study.

Consent for publication

All of the authors have agreed to publish this article in your journal if it should be accepted.

Competing interests

The authors declare that they have no competing interests.

\section{References}

1. Siegel RL, Miller KD, Jemal A. Cancer statistics (2019) CA Cancer J. Clin. 2019; 69: 7-34

2. Cannistra SA (2004) Cancer of the ovary. N Engl J Med 351:2519-2529

3. Geisler S, Coller J (2013) RNA in unexpected places: long non-coding RNA functions in diverse cellular contexts. Nat Rev Mol Cell Biol 14:699-712

4. Wilusz JE, Sunwoo H, Spector DL (2009) Long noncoding RNAs: functional surprises from the RNA world. Genes Dev 23:1494-1504 
5. Hung T, Chang HY (2010) Long noncoding RNA in genome regulation: prospects and mechanisms. RNA Biol 7:582-585

6. Cabili MN, Trapnell C, Goff L, Koziol M, Tazon-Vega B, Regev A et al (2011) Integrative annotation of human large intergenic noncoding RNAs reveals global properties and specific subclasses. Genes Dev 25:1915-1927

7. Ørom UA, Derrien T, Beringer M, Gumireddy K, Gardini A, Bussotti G et al (2010) Long noncoding RNAs with enhancer-like function in human cells. Cell 143:46-58

8. Katayama S, Tomaru Y, Kasukawa T, Waki K, Nakanishi M, Nakamura M et al RIKEN Genome Exploration Research Group, and Genome Science Group (Genome Network Project Core Group), and FANTOM Consortium. Antisense transcription in the mammalian transcriptome. Science 2005; 309 : 1564-1566

9. Wang JY, Lu AQ, Chen LJ (2019) LncRNAs in ovarian cancer. Clin Chim Acta 490:17-27

10. Braga EA, Fridman MV, Moscovtsev AA, Filippova EA, Dmitriev AA, Kushlinskii NE (2020) LncRNAs in Ovarian Cancer Progression, Metastasis, and Main Pathways: ceRNA and Alternative Mechanisms. Int J Mol Sci 21:8855

11. Zhan L, Li J, Wei B (2018) Long non-coding RNAs in ovarian cancer. J Exp Clin Cancer Res 37:120

12. Salamini-Montemurri M, Lamas-Maceiras M, Barreiro-Alonso A, Vizoso-Vázquez Á, RodríguezBelmonte E, Quindós-Varela M et al (2020) The Challenges and Opportunities of LncRNAs in Ovarian Cancer Research and Clinical Use. Cancers (Basel) 12:1020

13. Kel AE, Gössling E, Reuter I, Cheremushkin E, Kel-Margoulis OV, Wingender E (2003) MATCH: A tool for searching transcription factor binding sites in DNA sequences. Nucleic Acids Res 31:3576-3579

14. Liao Q, Liu C, Yuan X, Kang S, Miao R, Xiao H et al (2011) Large scale prediction of long non-coding RNA functions in a coding-non-coding gene co-expression network. Nucleic Acids Res 39:3864-3878

15. Pujana MA, Han JD, Starita LM, Stevens KN, Tewari M, Ahn JS et al (2007) Network modeling links breast cancer susceptibility and centrosome dysfunction. Nat Genet 39:1338-1349

16. Amelio I, Cutruzzolá F, Antonov A, Agostini M, Melino G (2014) Serine and glycine metabolism in cancer. Trends Biochem Sci 39:191-198

17. Vander Heiden MG (2011) Targeting cancer metabolism: a therapeutic window opens. Nat Rev Drug Discov 10:671-684

18. Radosław Januchowski P, Zawierucha (2013) Małgorzata Andrzejewska, Marcin Ruciński, Maciej Zabel. Microarray-based detection and expression analysis of ABC and SLC transporters in drugresistant ovarian cancer cell lines. Biomed Pharmacother 67:240-245

19. Uprety D, Adjei AA (2020) KRAS: From undruggable to a druggable Cancer Target. Cancer Treat Rev 89:102070

20. Jones S, Anagnostou V, Lytle K, Parpart-Li S, Nesselbush M, Riley DR et al (2015) Personalized genomic analyses for cancer mutation discovery and interpretation. Sci Transl Med 283:283ra53 


\section{Figures}

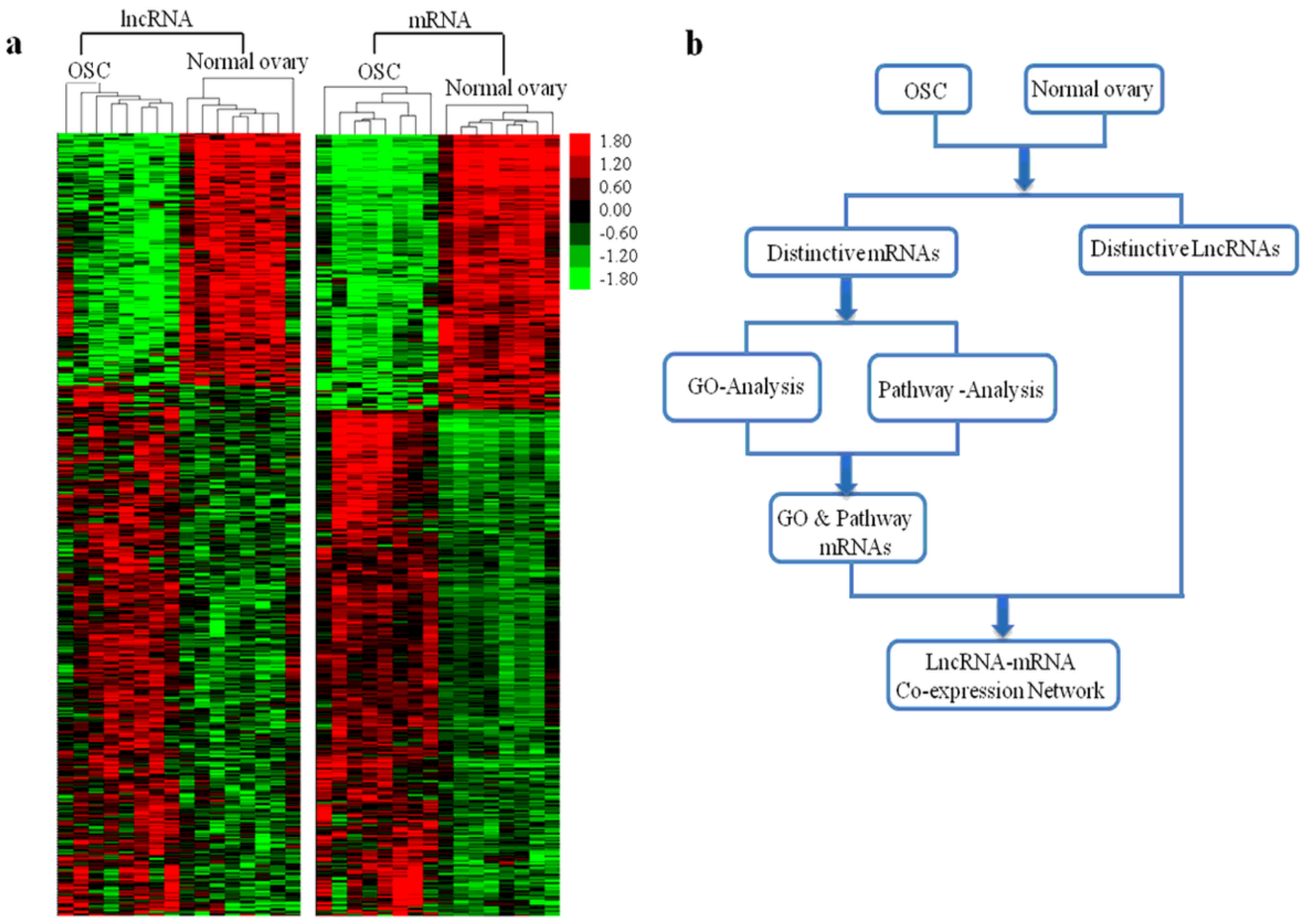

\section{Figure 1}

LncRNA and mRNA expression profile differences between OSC and normal ovary tissues. a: Hierarchical clustering of all samples revealed the non-random partitioning of samples into two major groups: one group containing eight OSC samples and another group containing eight normal ovary samples. Each column represents one sample, and each row represents one IncRNA or mRNA probe set. b: Schematic overview of the workflow for the construction of the IncRNA-mRNA co-expression network. 
a Up-regulated pathway

(Cellular Processes )

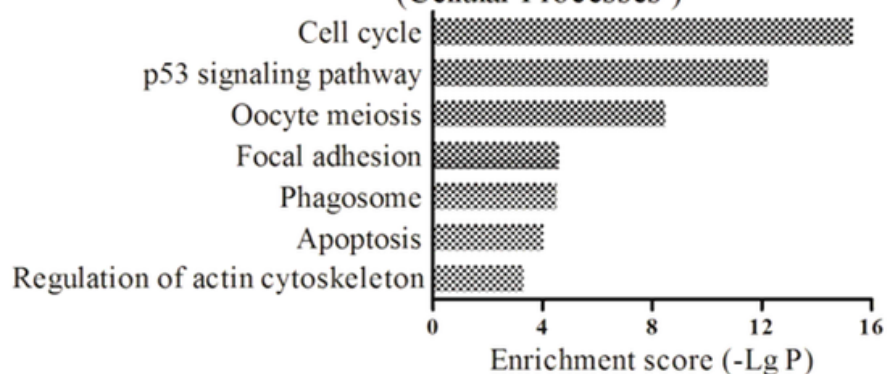

b

Up-regulated pathway

(Genetic Information Processing)

DNA replication

Mismatch repair man

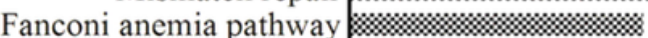

Homologous recombination …

Proteasome *

Ribosome biogenesis in eukaryotes \&

Nucleotide excision repair

Ubiquitin mediated proteolysis

RNA transport

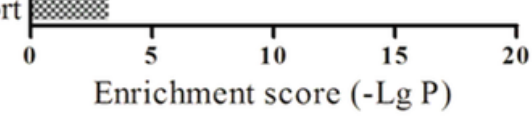

c Up-regulated pathway

(Environmental Information Processing)

ECM-receptor interaction * * .

VEGF signaling pathway

PI3K-Akt signaling pathway * * * :

HIF-1 signaling pathway m

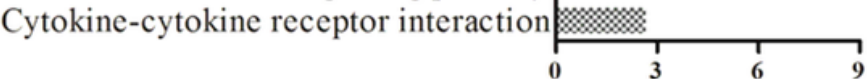

Enrichment score (-Lg P) d

Up-regulated pathway

(Immune system)

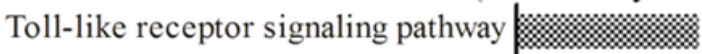

B cell receptor signaling pathway

Chemokine signaling pathway

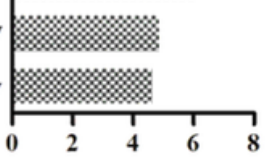

Enrichment score (-Lg P)

$\mathbf{e}$

Up-regulated pathway

( Human Diseases)

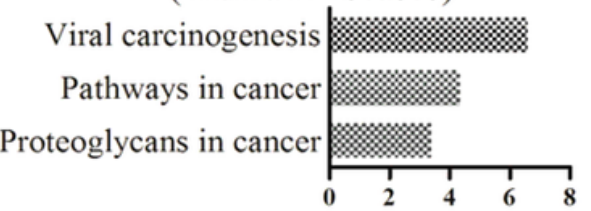

Enrichment score (-Lg P)

Up-regulated pathway

(Metabolism)

Tryptophan metabolism

Pyrimidine metabolism

Biosynthesis of amino acids

Purine metabolism

s.s.

Metabolic pathways

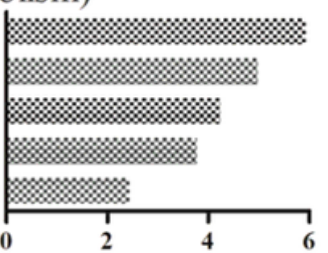

Enrichment score (-Lg P)

g Down-regulated pathway

(Metabolism/Environmental Information Processing)

Tyrosine metabolism

Drug metabolism-cytochrome P450 * * * * *

Ether Lipid metabolism ॥

$\mathrm{ABC}$ transporters

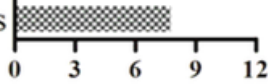

Enrichment score (-Lg P)

\section{Figure 2}

Functional predictions of the 67 IncRNAs in the IncRNA-mRNA co-expression network based on pathway analysis. Pathway analysis was applied to 109 mRNAs that were aberrantly expressed in OSC and coexpressed with 67 IncRNAs, which identified 32 upregulated pathways (involved in six biological processes) and five downregulated pathways $(P<0.05)$. 

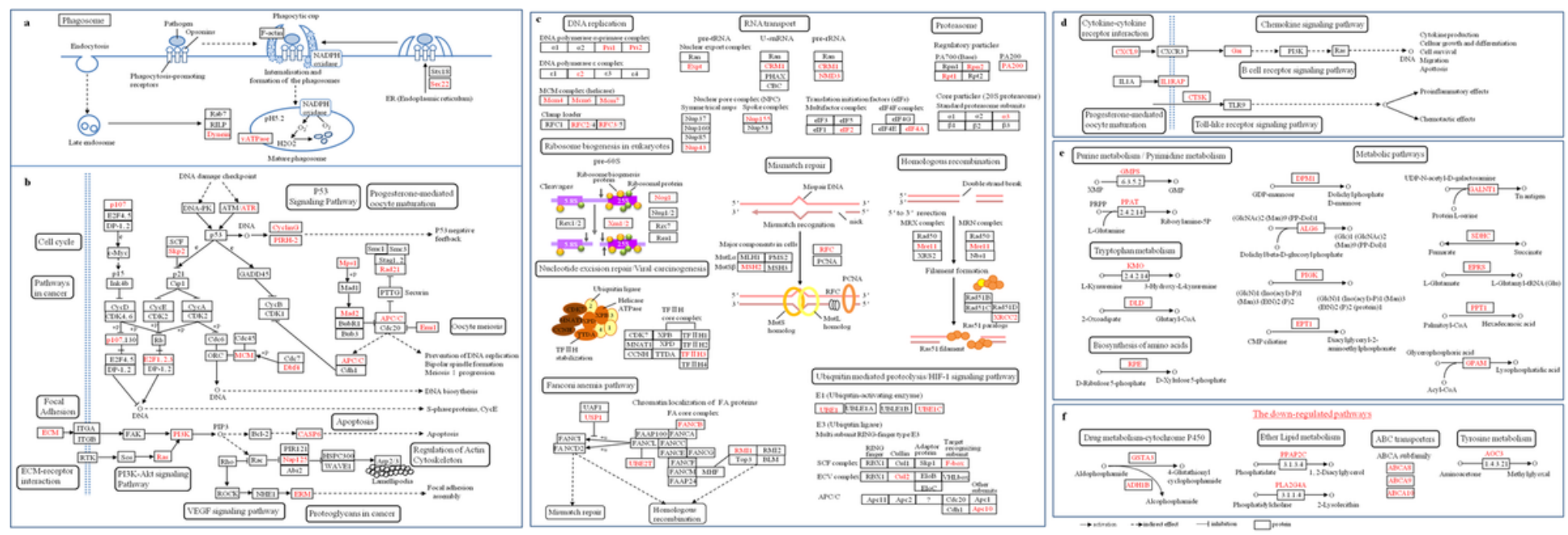

\section{Figure 3}

Schematic overview of the signaling pathways related to the 67 IncRNAs in the IncRNA-mRNA coexpression network. Red font represents the location of 82 mRNAs predicted by the co-expression network. Detailed descriptions of the IncRNAs and mRNAs are presented in Supplementary Table 10-16. 
a

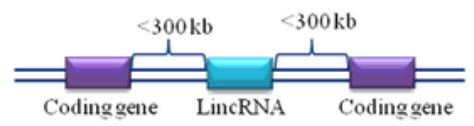

b

Up-regulated pathway
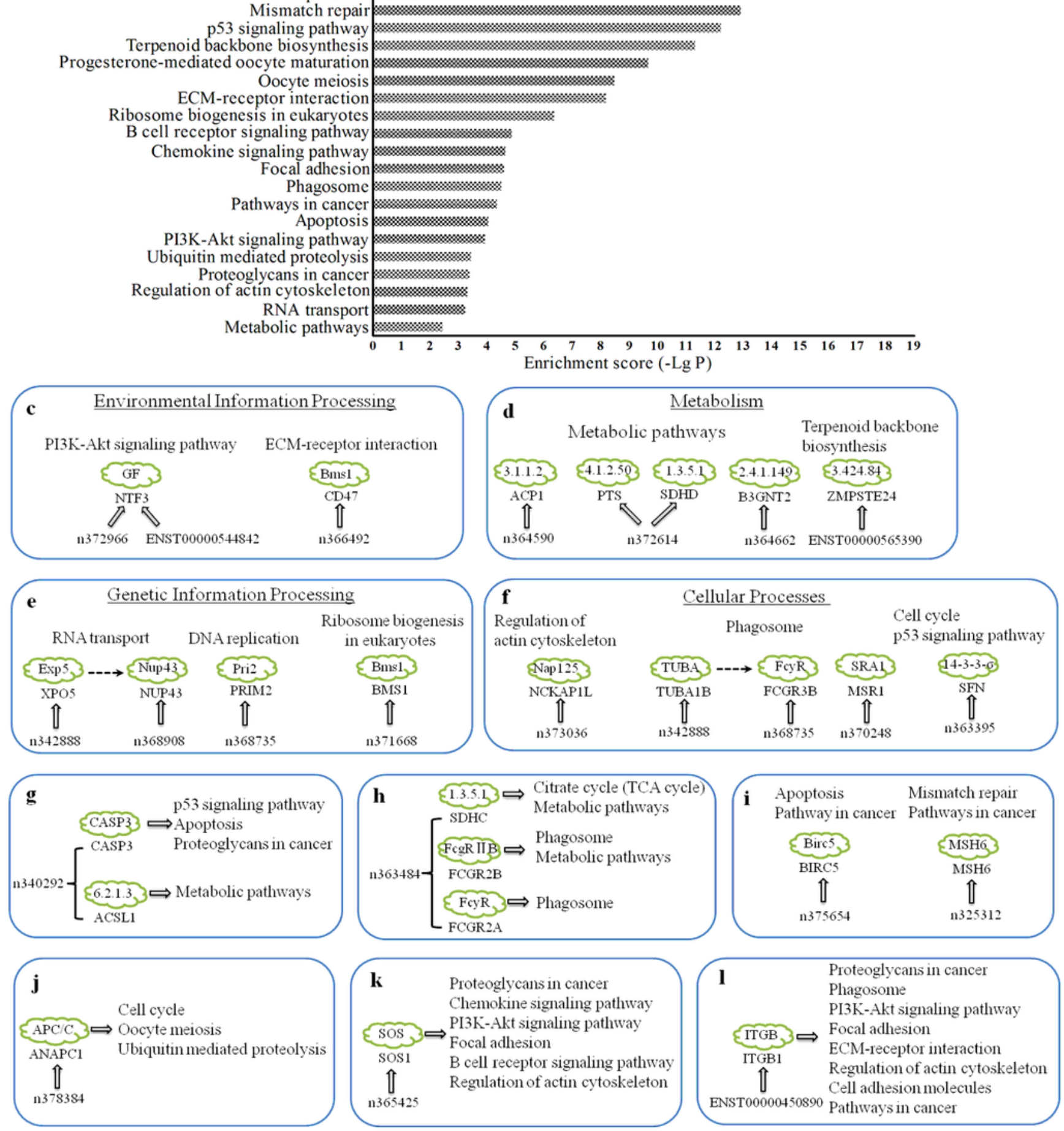

\section{Figure 4}

Functional predictions of the lincRNAs based on pathway analysis of adjacent coding genes within 300 $\mathrm{kb}$ in the genome. a: Schematic representation of a lincRNA. b: Pathway analysis was applied to 26 mRNAs adjacent to 23 lincRNAs, which identified 20 upregulated pathways related to tumorigenesis $(\mathrm{P}<$ 0.05). c-f: The 16 lincRNAs and adjacent coding genes were involved in four biological processes. G-I: The seven lincRNAs and adjacent coding genes were involved in multiple pathways. 
a

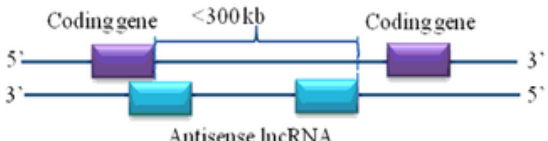

c

Down-regulated pathway ( Metabolism)

Metabolism of xenobiotics

by cytochrome $\mathrm{P} 450$

Purine metabolism

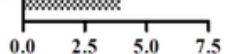

Enrichment score (-Lg P) b

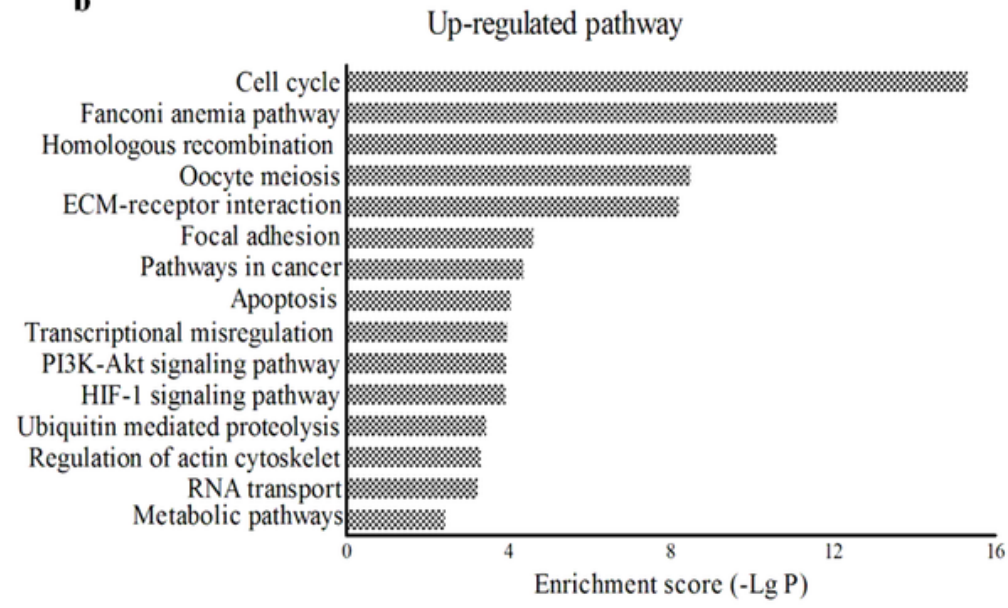

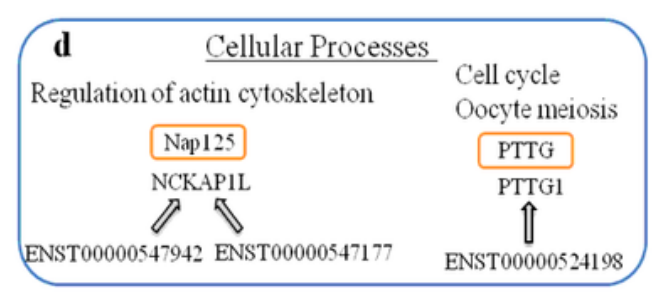

e

1.2 .1 .3

ALDH7A1

$\Uparrow$

ENST00000512500 ENST00000594870 ENST00000490375 ENST00000593382

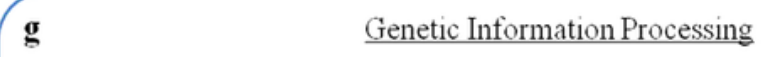

RNA transport Fanconi anemia pathway Homologous recombination

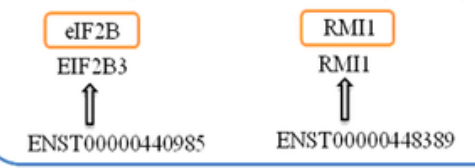

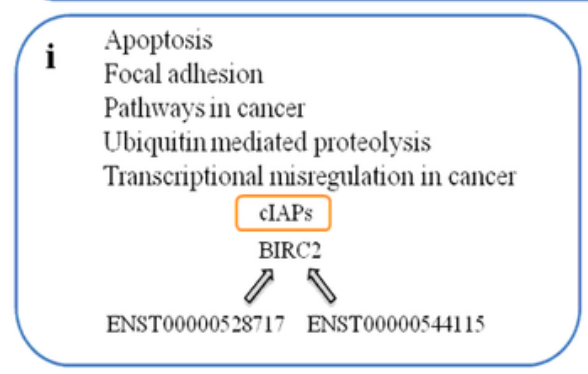

k

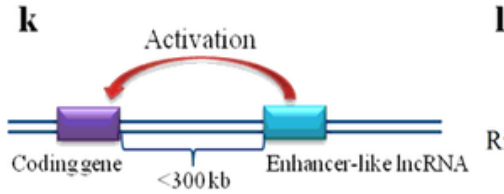
I

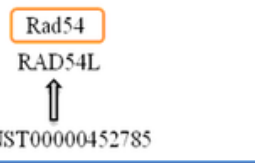

Fanconi anemia pathway Homologous recombination Pathways in cancer h

PI3K-Akt signaling pathway Focal adhesion ECM-receptor interaction Pathways in cancer

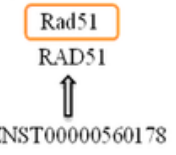

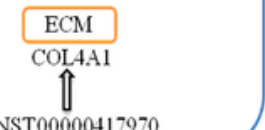

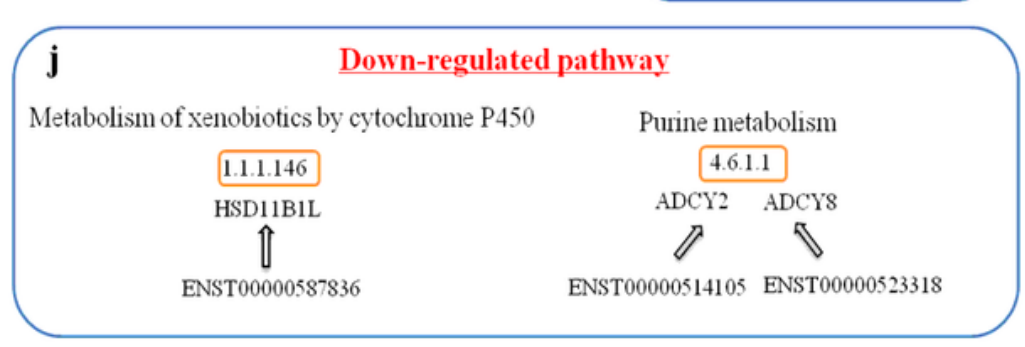

Ubiquitin mediated proteolysis Up-regulated pathway Cell cycle Oocyte meiosis

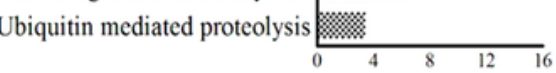
Enrichment score (-Lg P)

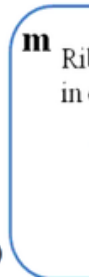

Cell cycle

Oocyte meiosis

Ubiquitin mediated proteolysis $\mathrm{APC} / \mathrm{C}$ ANAPCI $\Uparrow$ n378384

\section{Figure 5}

Functional predictions of the antisense IncRNAs according to pathway analysis of their sense coding genes, and the enhancer-like IncRNAs by pathway analysis of adjacent coding genes within $300 \mathrm{~kb}$ in the genome. a: Schematic representation of an antisense IncRNA. b-c: Pathway analysis was applied to 17 mRNAs that were sense coding genes of 19 antisense IncRNAs and were related to 15 upregulated pathways and 2 downregulated pathways $(P<0.05)$. d-i: The 16 antisense IncRNAs and their sense 
coding genes in upregulated signaling pathways. j: The three antisense IncRNAs and their sense coding genes in downregulated signaling pathways. k: Schematic representation of an enhancer-like IncRNA. I: Pathway analysis was applied to two mRNAs regulated by two enhancer-like IncRNAs and related to four upregulated pathways $(P<0.05)$. $\mathrm{m}$ : The two enhancer-like IncRNAs, two coding genes, and signaling pathways related to the coding genes.

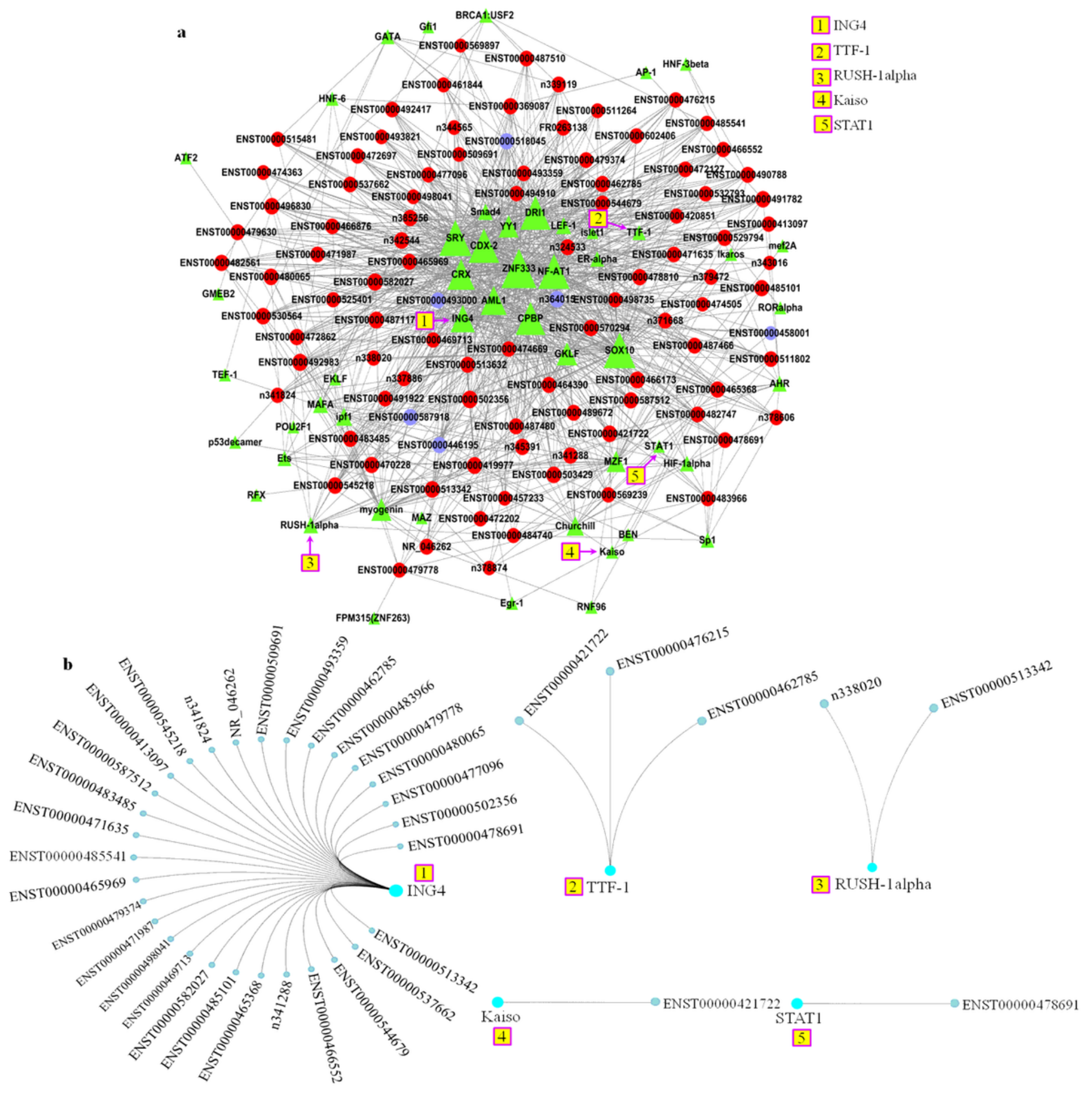

Figure 6 
Identification of TF-IncRNA relationships in OSC. a: Illustration of TF-IncRNA regulatory networks in OSC. b: The TFs ING4, TTF-1, RUSH-1 alpha, Kaiso, and STAT1 selectively regulated different target IncRNAs.

a

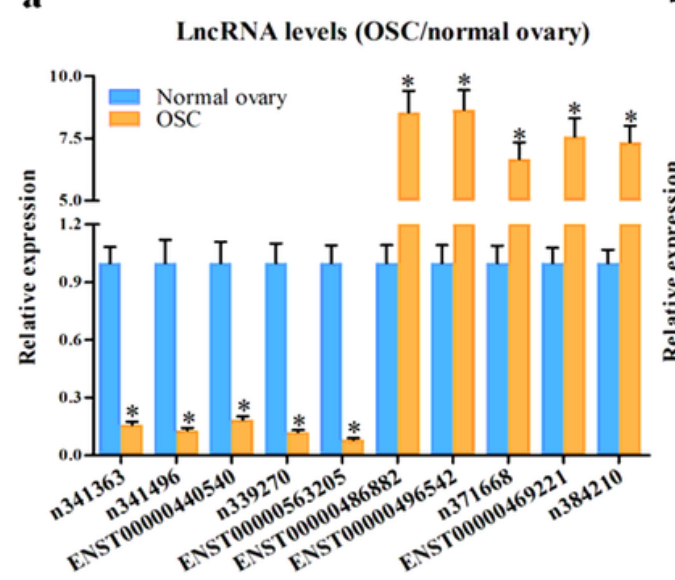

b

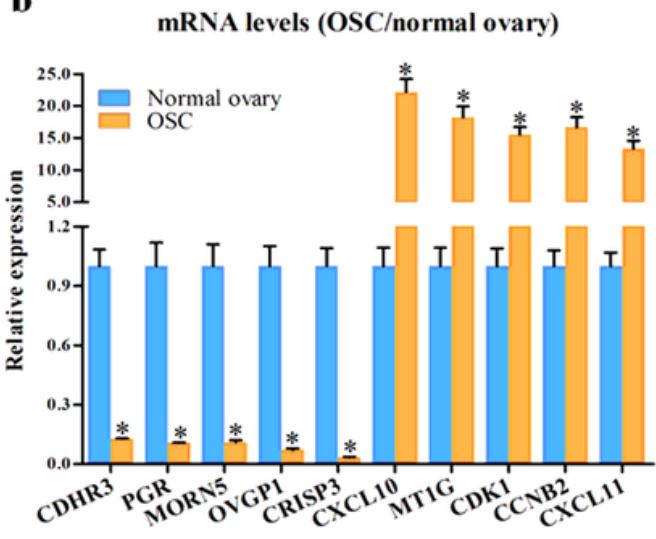

c

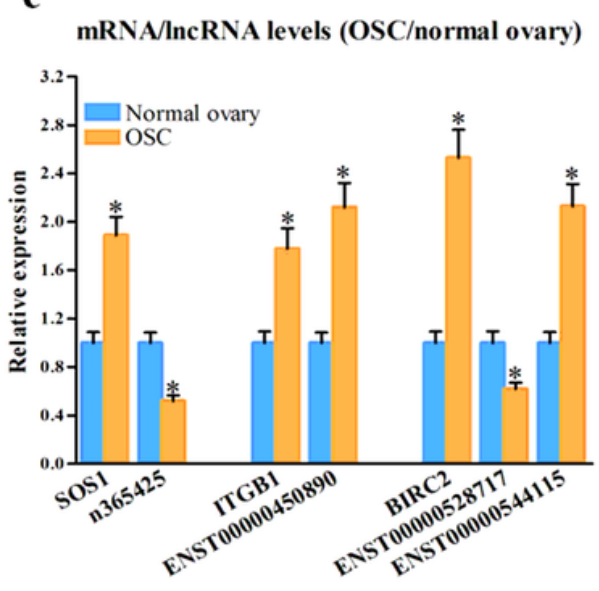

Figure 7

Expression analysis of IncRNAs and mRNAs in OSC tissues and normal ovary tissues. The expression level of a: five downregulated IncRNAs and five upregulated IncRNAs, b: five downregulated mRNAs and five upregulated mRNAs, c: SOS1 mRNA and n365425 IncRNA, ITGB1 mRNA and ENST00000450890, BIRC2 and ENST00000528717, ENST00000544115 IncRNAs were analyzed by qRT-PCR with the GAPDH gene as an internal control. Error bars represent the standard errors of independent samples. ${ }^{*} \mathrm{P}<0.05$.

\section{Supplementary Files}

This is a list of supplementary files associated with this preprint. Click to download.

- Fig.S1.png

- Fig.S2.png

- TableS1.docx

- TableS1016.docx

- TableS17.xlsx

- TableS18.xls

- Tables2.xlsx

- TableS4.xIsx

- TableS5.xlsx

- Tables6.xls

- Tables7.xls

- Tables8.xls

- Tables9.xls 